\title{
Encoding of event timing in the phase of neural oscillations
}

\author{
Anne Kösem ${ }^{\text {a,b,c }}$, Alexandre Gramfort ${ }^{\text {b,d,e }}$, Virginie van Wassenhove ${ }^{\text {a,b,c,* }}$ \\ a INSERM, U992, Cognitive Neuroimaging Unit, F-91191 Gif/Yvette, France \\ b CEA, DSV/I2BM, NeuroSpin Center, F-91191 Gif/Yvette, France \\ c Univ Paris-Sud, Cognitive Neuroimaging Unit, F-91191 Gif/Yvette, France \\ d INRIA, Parietal team, Saclay, F-91191 Gif-sur-Yvette, France \\ e LNAO, NeuroSpin, CEA Saclay, F-91191 Gif-sur-Yvette, France
}

\section{A R T I C L E I N F O}

\section{Article history:}

Accepted 4 February 2014

Available online 13 February 2014

\section{Keywords:}

MEG

Oscillatory entrainment

Temporal order

Simultaneity

Internal clock

\begin{abstract}
A B S T R A C T
Time perception is a critical component of conscious experience. To be in synchrony with the environment, the brain must deal not only with differences in the speed of light and sound but also with its computational and neural transmission delays. Here, we asked whether the brain could actively compensate for temporal delays by changing its processing time. Specifically, can changes in neural timing or in the phase of neural oscillation index perceived timing? For this, a lag-adaptation paradigm was used to manipulate participants' perceived audiovisual (AV) simultaneity of events while they were recorded with magnetoencephalography (MEG). Desynchronized AV stimuli were presented rhythmically to elicit a robust $1 \mathrm{~Hz}$ frequency-tagging of auditory and visual cortical responses. As participants' perception of AV simultaneity shifted, systematic changes in the phase of entrained neural oscillations were observed. This suggests that neural entrainment is not a passive response and that the entrained neural oscillation shifts in time. Crucially, our results indicate that shifts in neural timing in auditory cortices linearly map participants' perceived AV simultaneity. To our knowledge, these results provide the first mechanistic evidence for active neural compensation in the encoding of sensory event timing in support of the emergence of time awareness.
\end{abstract}

@ 2014 Elsevier Inc. All rights reserved.

\section{Introduction}

While dedicated neural structures for time perception have been described (Buhusi and Meck, 2005; Coull et al., 2004; Harrington et al., 1998; Ivry and Schlerf, 2008; Morillon et al., 2009; Treisman et al., 1990; van Wassenhove, 2009; Wittmann, 2009, 2013), the encoding of sensory event timing has been proposed to result from the intrinsic dynamics of neural populations not necessarily dedicated to temporal processing (Johnston and Nishida, 2001; Karmarkar and Buonomano, 2007; van Wassenhove, 2009). For instance, the timing of a colored visual patch could be encoded in the dynamics of the neural population dedicated to the analysis of color (Karmarkar and Buonomano, 2007; Moutoussis and Zeki, 1997). In this non-dedicated view, the latency of neural responses could provide an index for event timing (Johnston and Nishida, 2001; Zeki and Bartels, 1998). Under this latency code hypothesis, timing mechanisms are based on the changes of neural routing delays in sensory areas coding for a specific sensory attribute (Moutoussis and Zeki, 1997; Zeki and Bartels, 1998). To date however, electroencephalographic (EEG) studies have reported little-to-no

\footnotetext{
* Corresponding author at: CEA.DSV.I ${ }^{2}$ BM.NeuroSpin - INSERM Cognitive Neuroimaging Unit, NeuroSpin MEG, Brain Dynamics group, Bât 145 Point Courrier 156, F-91191 Gif sur Yvette, France.

E-mail address: Virginie.van.Wassenhove@gmail.com (V.van Wassenhove).
}

correspondence between neural latencies and participants' perceived timing (McDonald et al., 2005; Vibell et al., 2007), and rather suggest that it is the phase of neural oscillations that plays a crucial role in the encoding of visual event timing (Chakravarthi and Vanrullen, 2012; Gho and Varela, 1988).

We here provide further evidence that the encoding of event timing is realized in the phase of neural oscillations (in auditory cortex). It is well known that distinct phases of low-frequency neural oscillations are associated with periods of high and low neural excitability (Buzsáki, 2010; Lakatos et al., 2008). These fluctuations have been shown to impose temporal constraints on the "what" of perception by modulating the perceptual detection threshold of various stimuli (Busch et al., 2009; Fiebelkorn et al., 2013; Henry and Obleser, 2012; Monto et al., 2008; Neuling et al., 2012). They have also been proposed to serve parsing and informational chunking of sensory information over time (VanRullen and Koch, 2003) notably for complex temporal structures such as speech (Giraud and Poeppel, 2012). Indeed, neural oscillations are known to be entrained to external rhythms (Rees et al., 1986; Regan, 1966) and this entrainment may allow the alignment of cortical processing to the timing of sensory events (Giraud and Poeppel, 2012; Schroeder and Lakatos, 2009). As such, this mechanism naturally provides a means for the brain to internalize external temporal regularities (Schroeder and Lakatos, 2009). In line with this proposal, the phase of low-frequency neural oscillations has been 
shown to reflect temporal expectancy or predictability of event timing (Stefanics et al., 2010). Here, we hypothesize that the brain could use oscillatory entrainment to establish a temporal reference frame and we thus ask whether the phase of entrained neural oscillations actually encodes the "when" of perception. Specifically, the preferred phase of oscillatory entrainment is known to be context-dependent (Besle et al., 2011; Gomez-Ramirez et al., 2011; Lakatos et al., 2008; Rees et al., 1986), suggesting that neural entrainment may not be a passive neural response. Additionally, preferential phases of entrained neural oscillations are subject-specific (Besle et al., 2011), making this neural index particularly well-suited for investigating the highly subjective and variable nature of time perception.

To test the specific hypothesis that the phase of an entrained neural oscillation directly informs on the variability of conscious timing, we transiently shifted participants' perceived timing using a lagadaptation paradigm (Fujisaki et al., 2004; Miyazaki et al., 2006; Vroomen et al., 2004). Fig. 1 provides an overview of the experimental paradigm. During the induced changes of perceived timing, participants' brain activity was recorded with magnetoencephalography (MEG). During a given lag-adaptation block, audiovisual stimuli were presented rhythmically to induce an entrainment of oscillatory activity in sensory cortices. Analysis of MEG data showed that the preferential phase of entrained neural oscillations shifted during adaptation. Crucially, phase shifts of neural oscillatory entrainment in auditory cortex mirrored individuals' perceived simultaneity.

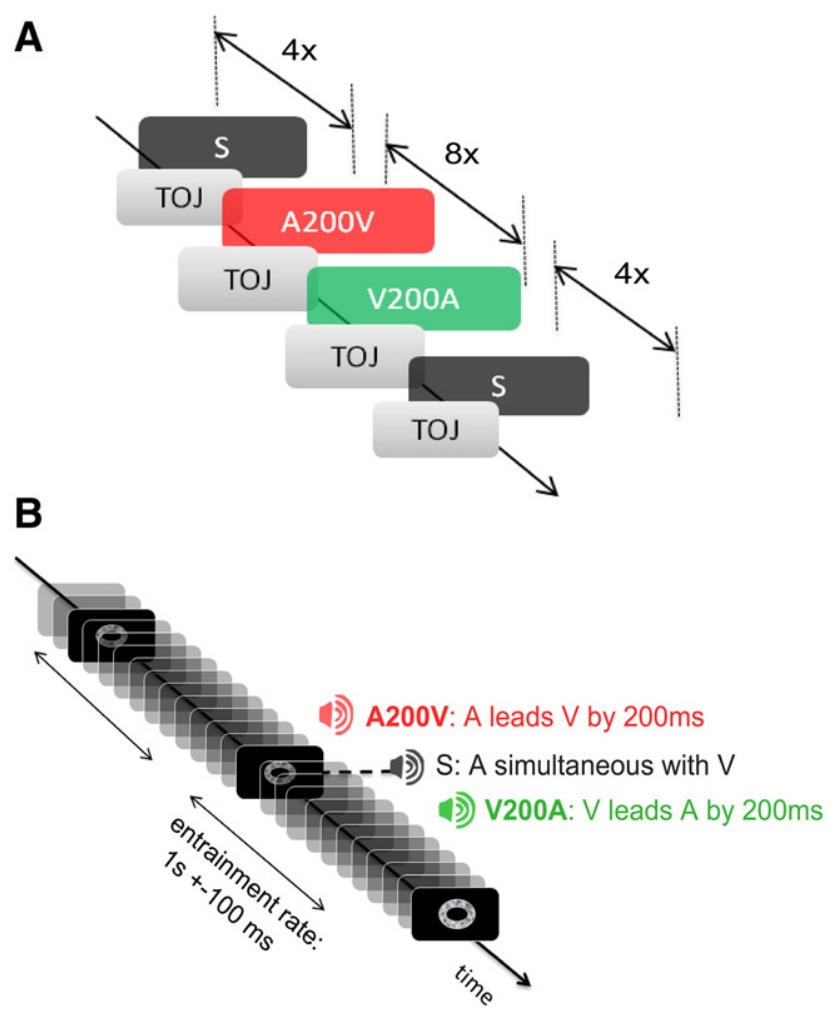

Fig. 1. Experimental design. (A) Three different audiovisual (AV) lag-adaptations were tested: simultaneous AV presentation (S, black), sound leading visual by $200 \mathrm{~ms}$ (A200V, red) and visual leading sound by $200 \mathrm{~ms}$ (V200A, green). Each lag-adaptation block was followed by a temporal order judgment (TOJ) block during which participants reported which of the auditory or visual event occurred first. One MEG session comprised eight blocks of each lag-adaptation (S, V200A and A200V). S blocks were run at the beginning and at the end of the MEG session; A200V and V200A alternated within the session. (B) In all lag-adaptation blocks, $65 \mathrm{AV}$ stimuli were presented at an average rate of $1 \mathrm{~Hz}$ with a random jitter of $+/-100 \mathrm{~ms}$. This experimental manipulation was designed to elicit neural entrainment at $1 \mathrm{~Hz}$ in sensory cortices.

\section{Materials and methods}

\section{Participants}

Nineteen participants ( 7 females, mean age: 24 years old) took part in the study. All had normal or corrected-to-normal vision, normal color vision and normal hearing, and were naive as to the purpose of the study. Each participant provided a written informed consent in accordance with the Declaration of Helsinki (2008) and the Ethics Committee on Human Research at NeuroSpin (Gif-sur-Yvette, France). Three subjects were excluded from the study: one subject did not finish the experiment, and two were unable to perform the temporal order judgment task properly. A total of sixteen participants were thus considered for MEG analyses.

\section{Stimuli}

The experiment was written in Matlab using the Psychophysics toolbox (Brainard, 1997). Visual stimuli consisted of disks lasting $16.7 \mathrm{~ms}$ ( 1 frame). A visual annulus ( $9.5^{\circ}$ of visual angle) consisted in the superposition of circles with different shades of gray. Visual stimuli were projected at a $60 \mathrm{~Hz}$ refresh rate onto a screen placed $90 \mathrm{~cm}$ away from participants seated under the MEG dewar. Auditory stimuli consisted of $16 \mathrm{~ms}$ duration white noise (incl. $5 \mathrm{~ms}$ fade-in and fade-out). Auditory stimuli were presented via Etymotic earphones (Etymotic Research Inc., USA).

\section{Procedure}

Two types of blocks were used in this experiment namely, lagadaptation ( 3 conditions: S, A200V or V200A) and temporal order judgment (TOJ) blocks. In the lag-adaptation blocks (Fig. 1B), a series of simultaneous (S) or desynchronized audiovisual events were displayed (A200V: audition leading vision by $200 \mathrm{~ms}$ or V200A: vision leading audition by $200 \mathrm{~ms}$ ). During the lag-adaptation block, a stream of $65 \mathrm{AV}$ stimuli was presented. The stream of AV events was displayed at an average rate of $1 \mathrm{~Hz}$; the stimulus onset asynchrony ( $\mathrm{SOA}$ ) between two successive auditory or visual stimuli was randomly chosen from a normal distribution with a mean of $1 \mathrm{~s}$ and a standard deviation of 100 ms: thus, each SOA has $95 \%$ probability to fall between $804 \mathrm{~ms}$ and $1196 \mathrm{~ms}$. The first $20 \mathrm{AV}$ events and the last $15 \mathrm{AV}$ events in the stream were made up of stimuli with a constant temporal lag. Three lags were tested: in the S condition, AV stimuli were synchronously displayed (lag $=0 \mathrm{~ms}$ ); in the A200V condition, the sound preceded the visual stimulus by $200 \mathrm{~ms}$ and in the V200A condition, the visual stimulus preceded the sound by $200 \mathrm{~ms}$. During the lag-adaptation block, participants were asked to count the number of temporal deviants that were introduced in the middle part of the lag-adaptation block. Temporal AV deviants consisted of desynchronized AV stimuli that deviated from the constant lag introduced at the beginning of the block. This task was introduced to insure that participants attended the temporal dimension of the AV stream which was reported to enhance temporal recalibration effects (Heron et al., 2010). Crucially however, only the first 20 and last $15 \mathrm{AV}$ stimuli are reported here namely the periods during which no temporal deviants were introduced. Each lag-adaptation block was systematically followed by a TOJ in which participants' subjective simultaneity of AV events was evaluated. In the TOJ blocks, AV stimuli were displayed with delays ranging from $+/-317,+/-217$, $+/-133,+/-67$, to 0 (a negative delay corresponds to the auditory leads and a positive delay corresponds to the visual leads). After each presentation of an AV pair, participants had to judge which of the sound or the visual event appeared first in a two alternative forced choice (2-AFC). Each condition was tested four times leading to a total of 36 trials in the TOJ blocks. The experiment started and ended with $4 \mathrm{~S}$ blocks (i.e. 4 times $\mathrm{S}+\mathrm{TOJ}$ ). Other blocks were alternated between 
A200V and V200A condition. In total, each condition was run in 8 lagadaptation + TOJ blocks leading to a total of 24 blocks (Fig. 1A).

\section{Data acquisition and preprocessing}

\section{MEG data acquisition}

Brain magnetic fields were collected in a magnetically shielded room using the whole-head Elekta Neuromag Vector View 306 MEG system (Neuromag Elekta LTD, Helsinki) equipped with 102 triplesensor elements (two orthogonal planar gradiometers and one magnetometer per location). Participants were seated in the upright position. Participants' head position was measured before each block with four head position coils (HPI) placed over the frontal and mastoid areas. Three fiducial points (nasion, left and right pre-auricular areas) and were used during the digitization procedure to help coregistration with anatomical MRI. MEG recordings were sampled at $1 \mathrm{kHz}$; bandpass filtered between $0.03 \mathrm{~Hz}$ and $330 \mathrm{~Hz}$ and used Maxshield. The electro-occulograms (EOG, horizontal and vertical eye movements) and electrocardiogram (ECG) were simultaneously recorded with MEG. Before each experiment, a so-called empty room recording of about 1 min with no subject sitting under the dewar was acquired for the computation of the noise covariance matrix.

\section{MEG data preprocessing}

Signal space separation (SSS) was applied to decrease the impact of external noise (Taulu et al., 2003). SSS correction, head movement compensation, and bad channel rejection were done using MaxFilter Software (Elekta Neuromag). Signal-space projection (SSP) was computed by principal component analysis (PCA) using Graph software (Elekta Neuromag) to correct for eye-blinks and cardiac artifacts (Uusitalo and Ilmoniemi, 1997). A rejection criterion for epochs was applied for gradiometers with amplitude exceeding $4000 \mathrm{e}^{-13} \mathrm{~T} / \mathrm{m}$.

\section{Structural MRI acquisition}

Magnetic resonance imaging (MRI) was used to provide highresolution structural image of each individual's brain. The anatomical MRI was recorded using a 3-T Siemens Trio MRI scanner. Parameters of the sequence were: voxel size: $1.0 \times 1.0 \times 1.1 \mathrm{~mm}$; acquisition time: $466 \mathrm{~s}$; repetition time $\mathrm{TR}=2300 \mathrm{~ms}$; and echo time $\mathrm{TE}=2.98 \mathrm{~ms}$.

\section{Anatomical MRI segmentation}

Volumetric segmentation of participants' anatomical MRI and cortical surface reconstruction was performed with the FreeSurfer software (http://surfer.nmr.mgh.harvard.edu/) (Dale et al., 1999; Fischl and Dale, 2000). These procedures were used for group analysis with the MNE suite software (http://www.martinos.org/mne/). Individuals' current estimates were registered onto the FreeSurfer average brain for surface based analysis and visualization.

\section{Co-registration procedure (MEG-aMRI)}

The co-registration of MEG data with the individual's structural MRI was carried out by realigning the digitized fiducial points with MRI slices. Using mne_analyze within the MNE suite, digitized fiducial points were aligned manually with the multimodal markers on the automatically extracted scalp of the participant. To insure reliable coregistration, an iterative refinement procedure was then used to realign all digitized points (about 30 more supplementary points distributed on the scalp of the subject) with the individual's scalp.

\section{Data analysis}

\section{MEG source reconstruction}

Individual forward solutions for all source locations located on the cortical sheet were computed using a 3-layers boundary element model (BEM) (Hämäläinen and Sarvas, 1989) constrained by the individual's anatomical MRI. Cortical surfaces extracted with FreeSurfer were sub-sampled to about 5120 equally spaced vertices on each hemisphere. The noise covariance matrix for each individual was estimated from the raw empty room MEG recordings preceding the individual's MEG acquisition. The forward solution, noise covariance and source covariance matrices were used to calculate the dSPM estimates (Dale et al., 1999). The inverse computation was done using a loose orientation constraint (loose $=0.2$, depth $=0.8$ ) (Lin et al., 2006). The cortically constrained reconstructed sources were then registered, morphed, onto the FreeSurfer average brain for group-level statistical analysis that was performed with MNE-python (Gramfort et al., 2013, 2014).

\section{Labels of interest}

We restricted the analysis to labels of interest in auditory and visual sensory cortices in the right hemisphere on the average FreeSurfer brain after morphing. Known hemispheric asymmetries in auditory cortex have consequences on the signal-to-noise ratio of MEG recordings across hemispheres (Shaw et al., 2013). Consistent with this, a great majority of our participants showed a higher and more reliable SNR in the right hemisphere. Labels were drawn individually based on the following two criteria: (i) maximal amplitude of the M100 response to auditory (resp. visual) stimulus for the auditory (resp. visual) label; and (ii) consistency with functional anatomy. Individuals' labels are presented in Fig. S2 superimposed on the FreeSurfer average brain.

\section{Event-related fields and source reconstruction}

Event-related fields (ERF) were computed by averaging 15 trials at the beginning and at the end of a lag-adaptation block. Data were gathered across the 8 lag-adaptation blocks for each asynchrony condition (S, A200V, V200A). For auditory ERF, the stimulus onset was locked to the sound onset; for visual ERF, the stimulus onset was locked to the visual stimulus. Data were segmented in epochs of $1 \mathrm{~s} \mathrm{(400} \mathrm{ms} \mathrm{pre-} \mathrm{and}$ $600 \mathrm{~ms}$ post-stimulus onset). Baseline correction was applied using the first $200 \mathrm{~ms}$ of the epoch ( -400 to $-200 \mathrm{~ms}$ pre-stimulus onset). The inverse solver used to localize the sources was then applied on the averaged normed evoked data. The normalization procedure was done to alleviate source cancellation when averaging sources within a label of interest, and across subjects (Gross et al., 2013). The comparisons of evoked responses between conditions were computed using a non-parametric permutation test. Correction for multiple comparisons was performed with cluster level statistics using as base statistic Student $t$-test computed at each time sample (Maris and Oostenveld, 2007). Only temporal clusters with corrected p-value $\leq 0.05$ are reported.

\section{Power spectrum analysis}

Low-frequency components in the frequency spectra could either originate from neural entrainment to the $1 \mathrm{~Hz}$ stimulation or from noise having a power spectrum density with $1 / \mathrm{f}$ distribution. To substantiate a peak neural entrainment at $1 \mathrm{~Hz}$, the $1 / \mathrm{f}$ component was removed by subtracting at each frequency bin the mean power of the neighboring frequency values ( 4 frequency values were: [fo $-0.14 \mathrm{~Hz}$; fo $-0.07 \mathrm{~Hz}$; fo $+0.07 \mathrm{~Hz}$; fo $+0.14 \mathrm{~Hz}]$ ) (Nozaradan et al., 2011).

\section{Phase analyses}

The first 5 trials of each lag-adaptation block were discarded as they constituted transient episodes before the establishment of the steadystate regime (Capilla et al., 2011). Hence, an equal number of 15 trials (or stimuli) at the beginning and at the end of a lag-adaptation period were considered for analysis. Single trial data were convolved with a 3-cycle Morlet wavelet centered at $1 \mathrm{~Hz}$ with a full width at half maximum of the power in the frequency domain of [0.7 Hz, $1.3 \mathrm{~Hz}$ ] (Keil et al., 2013) (Fig. 2). Epoch lengths were $4 \mathrm{~s}$ and centered on the visual or on the auditory stimulus onset. From the coefficients obtained with wavelet convolution the instantaneous phase at visual or auditory onset was extracted (as indicated in the text where relevant). Subsequent analyses were done on the distribution of phase values across 
A200V

- beginning of adaptation - end of adaptation

A
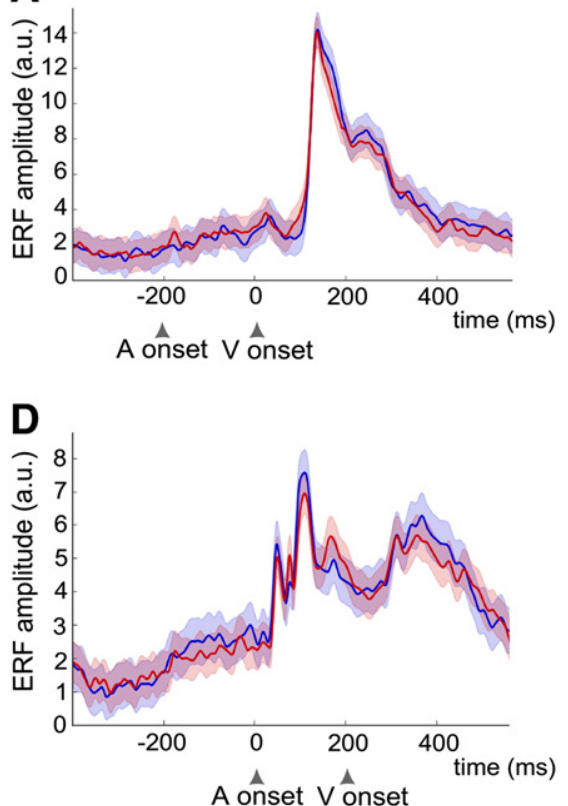

V200A

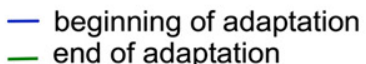

- end of adaptation
S

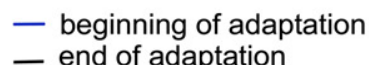

- end of adaptation
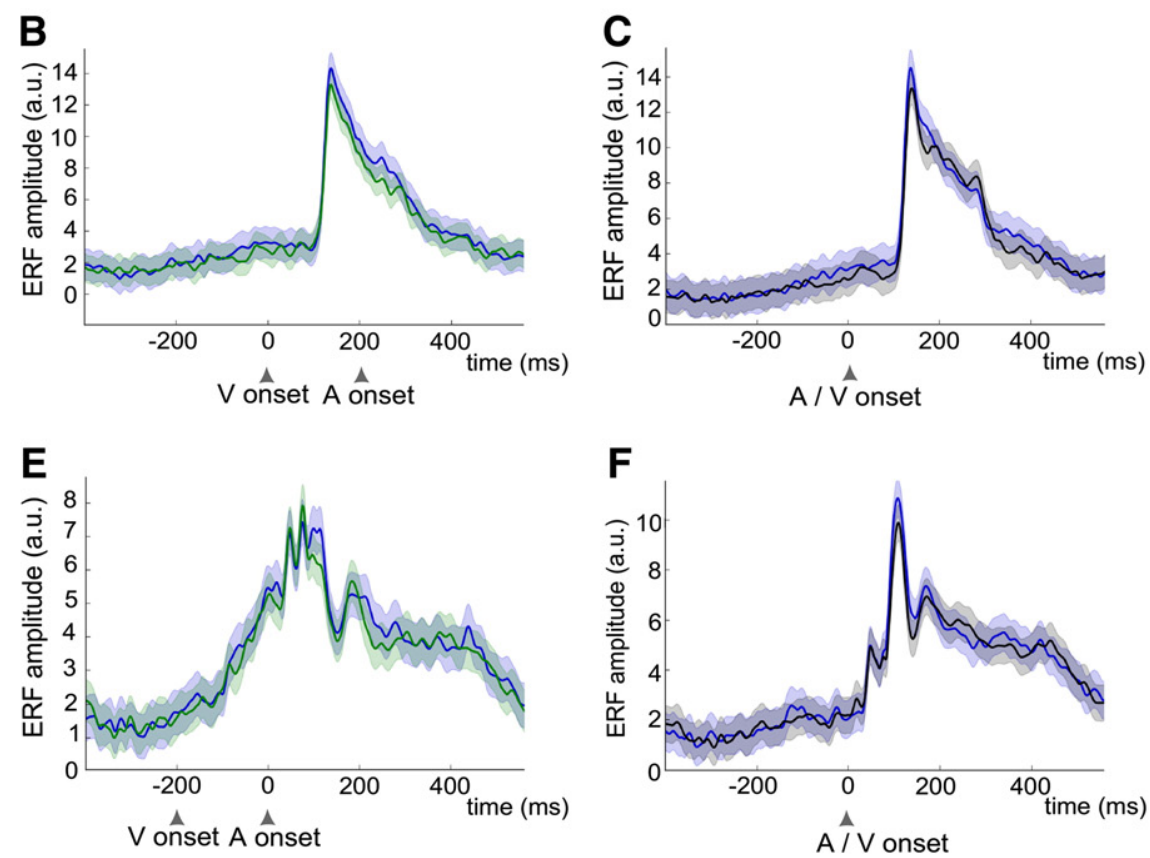

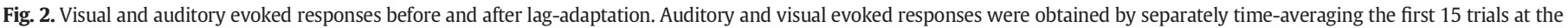

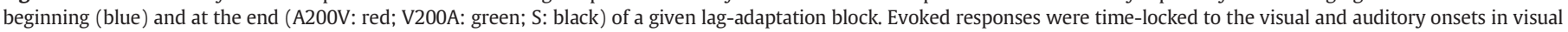

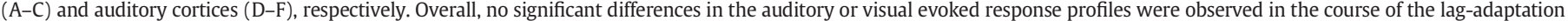

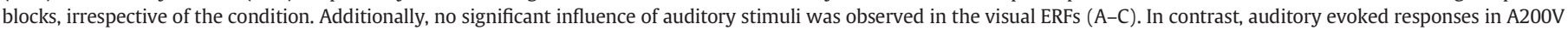

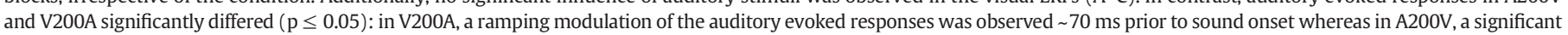
modulation of the auditory evoked responses at $\sim 300$ ms post-sound onset was observed.

trials gathered across specific condition, namely: the beginning or end of a lag-adaptation period, conditions S, A200V or V200A and for each participant.

\section{Phase uniformity test}

Phase distributions were submitted to Rayleigh's test for uniformity of phase data (Fisher, 1995). A significant Rayleigh test ( $\mathrm{p} \leq 0.05$ ) indicates that the distribution of phases shows a phase preference. If significance was reached, the circular mean of each distribution was computed and used for phase/ERFs and phase/behavior correlation analyses.

For each participant and each condition, a Rayleigh test was performed. If all three conditions passed the test, entrainment was considered to be true for that participant. When separating trials between the beginning and the end of a lag-adaptation period, a criterion of two out of three conditions passing the Rayleigh test was considered evidence for entrainment.

The phase-locking value (PLV) (Lachaux et al., 1999) is defined as:

$\operatorname{PLV}(t)=\frac{1}{K}\left|\sum_{k=1}^{K} \mathrm{e}^{\mathrm{j} \theta(t, k)}\right|$

where $K$ is the number of trials, and $\theta(t, k)$ is the instantaneous phase at time $t$ and trial $k$. PLVs were computed to assess intra-subject variability in the preferential phase.

\section{Statistical comparison of phase distributions}

To assess statistical significance of phase shifts between 2 conditions (e.g. A and B), bootstrap measure of $95 \%$ confidence interval was used on the phase distribution of the paired differences A-B (Fisher, 1995).
Phase distributions A and B were statistically different if the mean of the difference was statistically different from zero, i.e. if zero lies outside the measured confidence interval ( $\mathrm{p} \leq 0.05)$.

Psychophysics - point of subjective simultaneity

The percentage of "visual first" responses during the TOJ task following the lag-adaptation period (S, V200A, and A200V) were plotted as a function of AV asynchrony and fitted with a logistic regression to a sigmoid function of the form:

$$
\text { fit }=\frac{1}{1+\exp \left(\frac{\text { data }- \text { PSS }}{\text { JND }}\right)} \text {. }
$$

From each individual fit, the point of subjective simultaneity (PSS) value and the just noticeable difference (JND) were estimated. The PSS corresponds to the AV asynchrony at which an individual responds at chance level (50\%) in a TOJ task and thus taken as a true subjective simultaneity estimate (Vroomen and Keetels, 2010).

\section{Results}

Participants underwent a series of alternating lag-adaptation and TOJ blocks while being recorded with MEG. Three AV delays were tested (Figs. 1A-B): simultaneous AV presentations (S, control condition), a sound leading a visual event by $200 \mathrm{~ms}$ (A200V) and a visual event leading a sound by $200 \mathrm{~ms}$ (V200A). Each TOJ block allowed establishing an individual's psychometric curve following each lag-adaptation block as well as deriving the progression of the individual's point of subjective simultaneity (PSS). Our hypothesis was that changes in neural activity during lag-adaptation would predict changes in subjects' perceived 
simultaneity. First, we tested the latency code hypothesis by comparing the event-related responses at the beginning and at the end of the lagadaptation. We then tested the phase code hypothesis by comparing the phase of the entrained neural oscillation at the beginning and the end of the adaptation (Supplementary Fig. S1).

\section{Stable evoked activity in sensory cortices during adaptation}

Auditory and visual event-related fields (ERFs) were sourcereconstructed. The resulting time-source series were separately averaged in the auditory and visual labels. In all three lag-adaptation conditions, visual evoked responses were comparable with no significant modulations of the visual evoked responses by auditory stimuli (Figs. 2A-C). To the contrary, clear modulations of the auditory evoked responses by the presence of visual stimuli were observed (Figs. 2D-F) in both A200V and V200A. The auditory evoked response profiles in A200V and V200A significantly differed ( $\mathrm{x} \leq 0.05)$ : in V200A, a ramping modulation of the auditory evoked response was observed $\sim 70 \mathrm{~ms}$ prior to sound onset whereas in A200V, a significant modulation of the auditory evoked response at $\sim 300 \mathrm{~ms}$ post-sound onset was observed. The modulations of the auditory evoked responses remained steady throughout the lag-adaptation block and did not significantly differ between the beginning and the end of a given lag-adaptation block. If, as hypothesized, changes in perceived timing were caused by changes in the neural timing of auditory and visual cortices during lag-adaptation, the latency of evoked activity did not appear to be a good candidate to capture this change. These observations are in agreement with previous findings on evoked-related-potential literature (McDonald et al., 2005).

Non-stationarity of the entrained neural oscillations during lag-adaptation

As predicted by the rate of AV stimulation during lag-adaptation, neural activity over long time scales displayed periodic fluctuations at $1 \mathrm{~Hz}$ i.e. oscillatory entrainment or frequency-tagging: a characteristic frequency peak at $1 \mathrm{~Hz}$ was clearly observable in auditory and visual power spectra (Figs. 3B, D) and in single-trial data (Figs. 3A, C). No significant changes in $1 \mathrm{~Hz}$ power were found between the beginning and the end of a given lag-adaptation period irrespective of the experimental condition.

Additionally, $1 \mathrm{~Hz}$ oscillatory activity showed a significant phaselocking in both sensory cortices. Phase preferences of the $1 \mathrm{~Hz}$ oscillation were tested using a Rayleigh test against uniformity $(\mathrm{p}<0.05)$. At the beginning of all lag-adaptation periods (S, A200V, and V200A), phase preferences were found to be significant in both sensory cortices for 15 participants; at the end of all lag-adaptation periods (S, A200V, and V200A), significant phase preferences were found for all participants. For all conditions and in both sensory cortices, the observed phase locking values (PLV, index of the variance of phase distributions) did not significantly differ between the beginning and the end of each lag-adaptation period (Supplementary Table S1). Altogether, these results show the existence of robust phase preferences in all conditions throughout the course of the experiment.

However, and unlike the power of the entrained $1 \mathrm{~Hz}$ oscillation, the phase of the $1 \mathrm{~Hz}$ oscillatory component did not appear to be stationary over the course of the lag-adaptation period. Specifically, the neural responses evoked by the stimulus presentation arrived at different phases of the $1 \mathrm{~Hz}$ oscillation (Figs. 4A, B). In the non-zero lag adaptations (A200V and V200A), phases of the $1 \mathrm{~Hz}$ oscillation shifted in opposite directions in visual and auditory cortices whereas in the control condition (S), no phase shifts were observed (Fig. 4C). Within a given block, stimuli presented at the beginning of the adaptation were identical to those presented at the end: as previously reported, and consistent with the steady stimulation, no significant differences were observed when contrasting the evoked responses at the beginning and at the end of a given lag-adaptation period (Fig. 2). If neural entrainment was a passive neural response, no changes in the preferential phase would be predicted. Hence, the observed phase shifts in the entrained oscillatory response suggest an active modulation of the entrained 1 $\mathrm{Hz}$ oscillation not easily accounted for by the unchanged event-related responses.

Additional analyses were performed supporting the independence of evoked activity and neural oscillatory phase shifts. If the evoked responses impacted the phase of neural oscillations at $1 \mathrm{~Hz}$, a similar pattern of phase shifts in neighboring frequency regions should be found by virtue of evoked response being fixed-latencies and strongly phaselocked signals. Weak-to-no phase locking and no significant phase shifts were observed for 2 or $3 \mathrm{~Hz}$ neural oscillations (Supplementary Fig. S2).

\section{Encoding of subjective timing in the phase of neural oscillations}

Our main hypothesis states that shifts in the phase of neural oscillations during adaptation may reflect active changes in subjective timing. To test this, we compared the shifts in the phase of neural oscillations with perceptual reports (Figs. 5A, B). Consistent with previous reports on TOJ paradigms (Love et al., 2013; Van Eijk et al., 2008), the average PSS value in the zero-lag adaptation condition (S) was biased towards sound-leading asynchronies: on average, participants required the auditory event to lead the visual event by $38 \mathrm{~ms}$ to consider them as simultaneous (Fig. 5B). Following lag-adaptation to A200V and V200A, participants required the sound to lead the visual event even more with PSS values of $-87 \mathrm{~ms}$ (significant main effect of lag-adaptation: $\mathrm{F}(2,30)=10.1, \mathrm{p}<0.001$, significant contrast $\mathrm{PSS}_{\mathrm{A} 200 \mathrm{~V}}-\mathrm{PSS}_{\mathrm{S}: \mathrm{p}}=$ $0.002)$ and $-69 \mathrm{~ms}\left(\mathrm{PSS}_{\mathrm{V} 200 \mathrm{~A}}-\mathrm{PSS}_{\mathrm{S}} \mathrm{p}=0.03\right)$, respectively (Fig. 5B). Thus, in both A200V and V200A, the sound needed to be heard before the visual event to be perceived as simultaneous, but shifts in perception were more pronounced in A200V than in V200A $\left(\mathrm{PSS}_{\mathrm{A} 200 \mathrm{~V}}-\mathrm{PSS}_{\mathrm{S}} \mathrm{p}=0.01\right)$. From a neural processing point of view, these results suggest that auditory analysis may be delayed during lag-adaptation and/or visual analysis advanced in time. As seen in Fig. 4C, the average phase shifts during A200V and V200A lagadaptations were consistent with these predictions: lag-adaptation leads to a negative shift of the phase of auditory entrained oscillation i.e. the $1 \mathrm{~Hz}$ response in auditory cortex shifted forward in time; conversely, the phase of the visual entrained oscillation positively shifted suggesting that the $1 \mathrm{~Hz}$ entrained response shifted backward in time.

It is noteworthy that while the direction of the PSS shifts observed after lag-adaptation to A200V was consistent with seminal reports on temporal recalibration (Fujisaki et al., 2004; Vroomen et al., 2004), the PSS following V200A lag-adaptation did not shift in the direction predicted by seminal temporal recalibration effects. Nevertheless, the latter finding remains consistent with other lag-adaptation reports (Miyazaki et al., 2006; Yamamoto et al., 2012) and major differences in experimental design including the choice of task and the absence of readapting trials in the TOJ assessment task could account for these differences (Cai et al., 2012; Yamamoto et al., 2012). Specifically, remote and recent stimulation histories are known to bias in opposite ways the perception of incoming stimuli (Chopin and Mamassian, 2012) and consistent with this, PSS shifts are distinctly influenced by the presence or the absence of re-adapting trials (Cai et al., 2012). Here, we did not include re-adapting trials during the TOJ blocks and stimuli were randomly chosen between $-316 \mathrm{~ms}$ and $+316 \mathrm{~ms}$, hence the primary influence on lag adaptation was the remote stimulation history i.e. the lag-adaptation trials.

Here, we used lag-adaptation to generate shifts in an individual's subjective timing. As expected, a large inter-individual variability was observed in the individual's default PSS (control S, zero-lag adaptation) and in the propensity of an individual to temporally adapt to desynchronized AV stimuli (Figs. 5A, C). In order to test whether the shifts in the phase of the $1 \mathrm{~Hz}$ neural oscillation were commensurate with subjective simultaneity, we capitalized on this inter-individual variability and compared individuals' PSS with the shifts in the entrained neural oscillation in each sensory cortex. Strikingly, the 


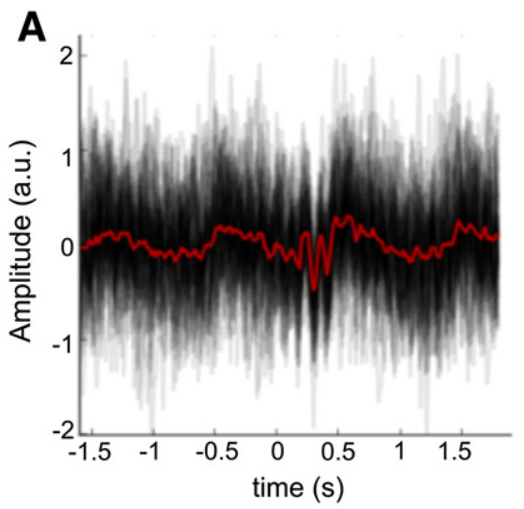

B
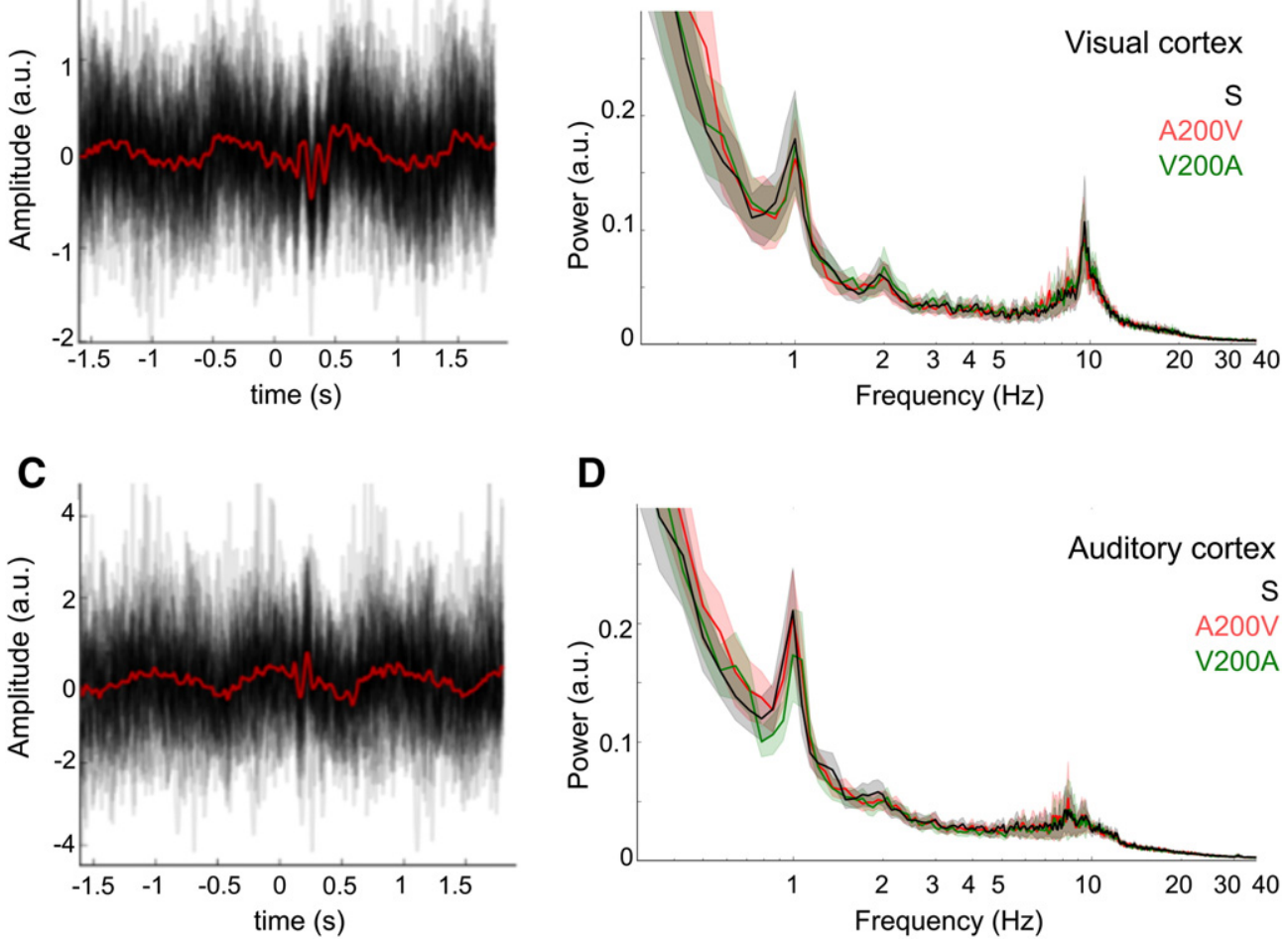

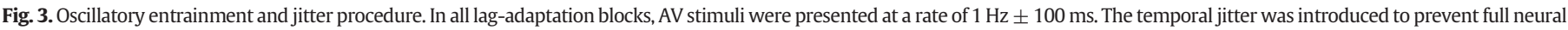

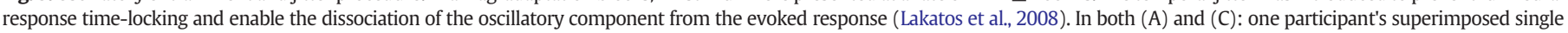

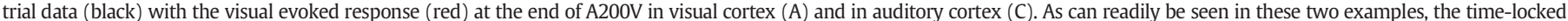

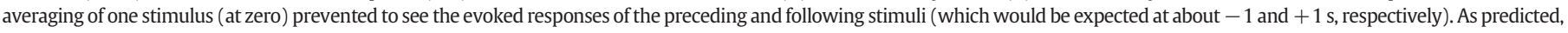

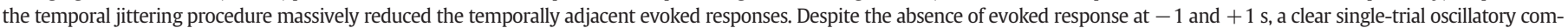

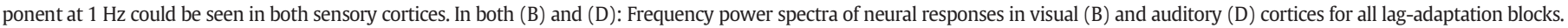

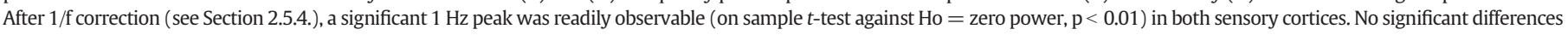

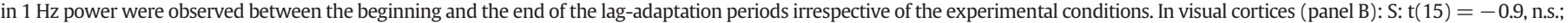
A200V: $\mathrm{t}(15)=0.6$, n.s.; and V200A: $\mathrm{t}(15)=-0.7$, n.s.. In auditory cortices (panel D): $\mathrm{S}$ : $\mathrm{t}(15)=-1.8, \mathrm{n} . \mathrm{s}$.; $\mathrm{A} 200 \mathrm{~V}$ : $\mathrm{t}(15)=0.05$, n.s.; V200A: $\mathrm{t}(15)=1.7$, n.s..

phase shifts of the $1 \mathrm{~Hz}$ auditory oscillatory neural response significantly correlated with participants' subjective simultaneity whereas no such correlations were observed in visual cortices (Fig. 6). Specifically in auditory cortex, the more negative the phase of $1 \mathrm{~Hz}$ neural oscillation, the more the sounds needed to lead visual events to be perceived as simultaneous. Thus a negative shift in phase, which corresponds to a forward neural timing i.e. a delay of $1 \mathrm{~Hz}$ auditory activity in time, is associated with a shift in perceived simultaneity towards auditory-lead asynchronies. Note that shifts in perception and shifts in neural timing change together in coherent directions: a delay in auditory processing as measured by a negative phase shifts should correspond to a perceived delay in auditory event timing; thus to perceive simultaneity the sound needs to be advanced in time i.e. the PSS is shifted towards auditorylead asynchronies. Conversely, a positive phase shift moves the processing of auditory events backward in time thereby sounds have to lag visual events to be perceived as simultaneous. As reported in Fig. 6, the slope of the regression between perceived timing shift and neural timing shift was 1.2, suggesting that neural timing and perceived timing shifts are quantitatively similar. Additionally, the obtained regression predicts that a zero-phase shift in the $1 \mathrm{~Hz}$ neural oscillation observed in auditory cortex (i.e. stationarity or stable phase preference through time) should map onto a PSS of $-45 \mathrm{~ms}$. This value was very close to the mean PSS obtained experimentally in the control condition S (namely, $-38 \mathrm{~ms}$ ).

Overall these results suggest that AV simultaneity relies on asymmetrical cross-talks between auditory and visual sensory cortices, namely: auditory cortices actively adjust the timing of auditory events to match that of visual inputs.

\section{Discussion}

Shifts in perceived AV simultaneity following lag-adaptation (Fujisaki et al., 2004; Miyazaki et al., 2006; Vroomen et al., 2004; Yamamoto et al., 2012) have been hypothesized to originate from mechanisms capable of adjusting the neural processing time across sensory modalities (Fujisaki et al., 2004; Moutoussis and Zeki, 1997; Stone et al., 2001; Sugita and Suzuki, 2003; Zeki and Bartels, 1998). In support of this hypothesis, our study reveals that such mechanisms may be implemented as phase shifts of neural oscillations: contrasting the sensory responses before and after AV lag-adaptation provided no evidence for a latency code hypothesis and instead revealed significant phase shifts of the entrained $1 \mathrm{~Hz}$ neural oscillations. Crucially, it is the phase shifts of the auditory response that linearly predicted participants' shifts of subjective AV simultaneity and no systematic mapping was found between visual responses and subjective AV timing. The present findings thus suggest that auditory cortex temporally calibrates its window of analysis with respect to vision and that event timing linearly maps onto the phase of entrained neural oscillations.

\section{Neural oscillations as pacemakers for the encoding of time}

The "internal clock" is a prominent model of time perception which is classically composed of a pacemaker (ticking mechanism), an accumulator (of ticks) and a counter (Church, 1984; Treisman, 1963). Of particular interest here, the pacemaker consists of an oscillator ticking at a frequency that can be modulated depending on the temporal properties of sensory stimuli (Buhusi and Meck, 2009; Treisman, 1984; Treisman 

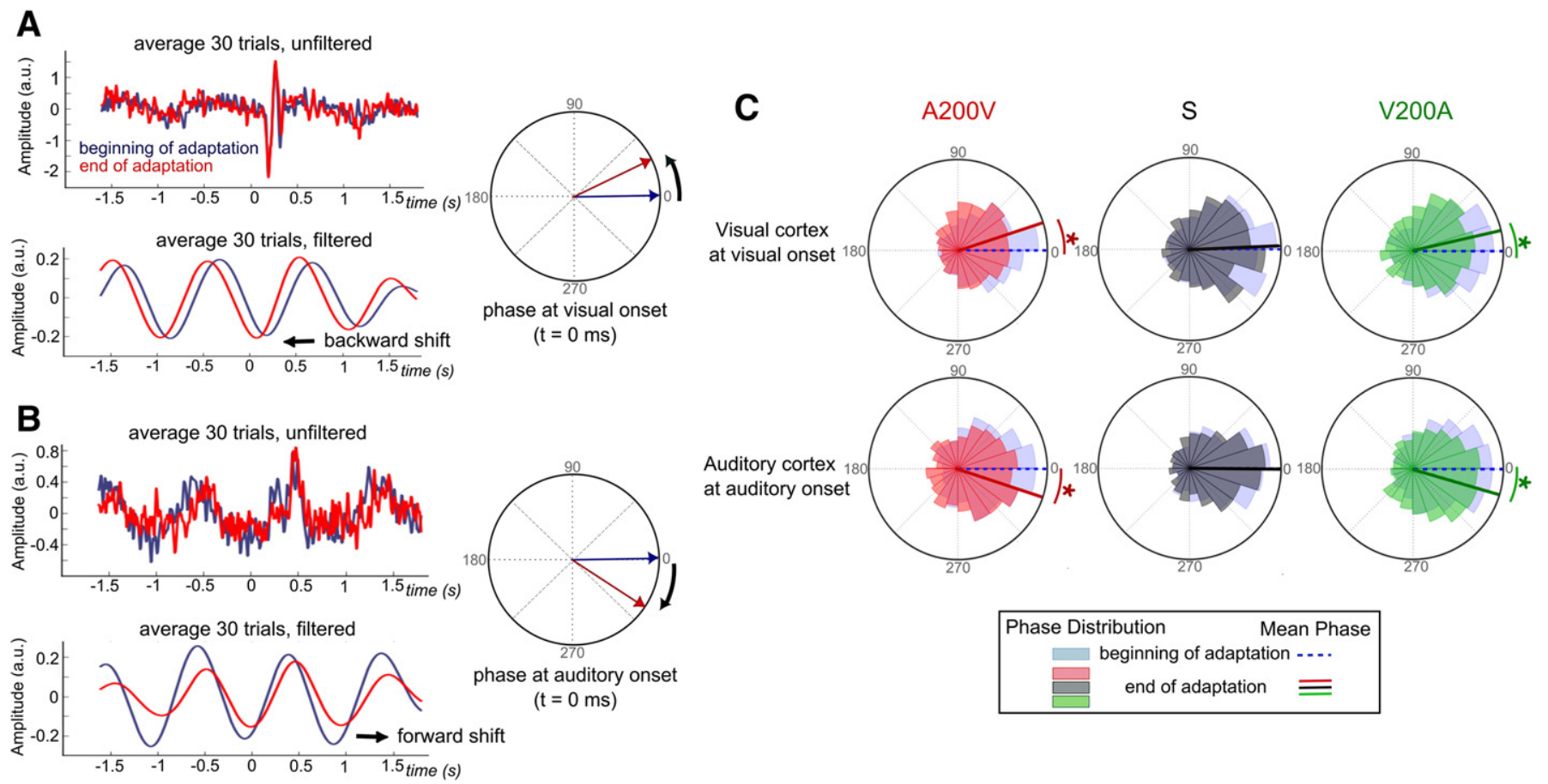

$(\mathrm{t}=0 \mathrm{~ms})$

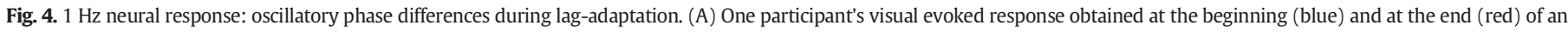

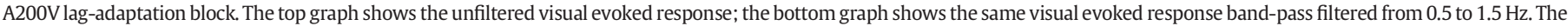

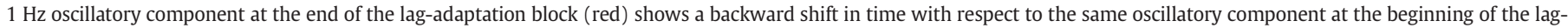

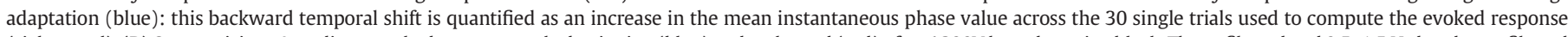

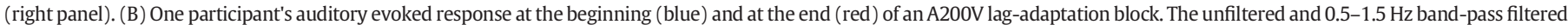

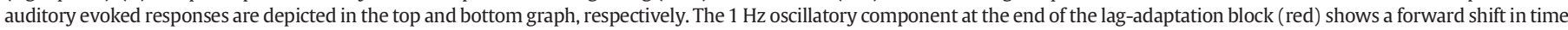

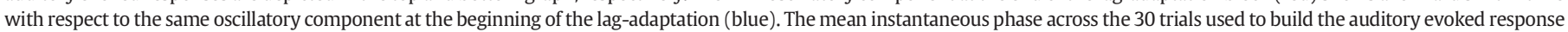

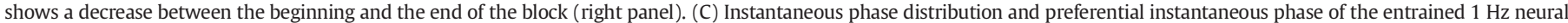

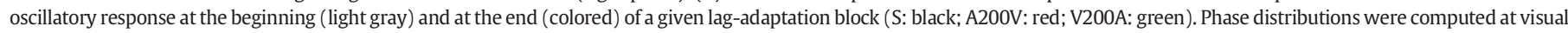

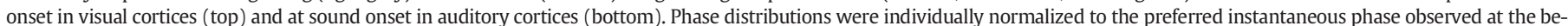

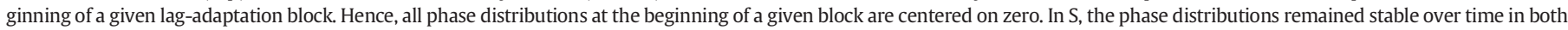

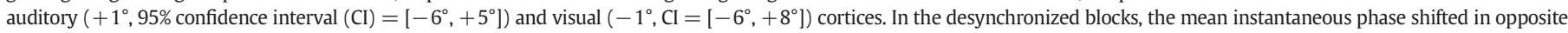

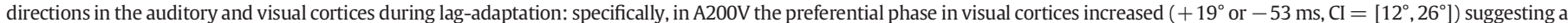

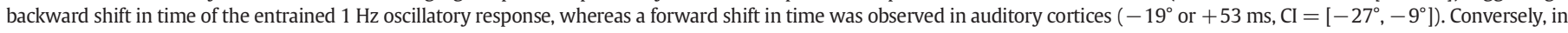

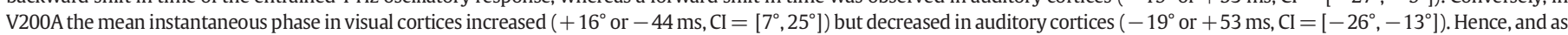

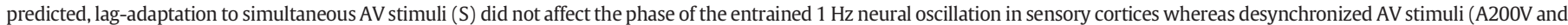
V200A) shifted the preferential phase distribution in opposite direction in the auditory and visual cortices.

et al., 1990, 1992): specifically, external temporal regularities can impose modulations of the pacemaker frequency so as to entrain the internal clock (Treisman et al., 1992). Similarly, intrinsic neural oscillations match the temporal scales of perceptual phenomena (Buzsáki and Draguhn, 2004; Roopun et al., 2008; van Wassenhove, 2009; Wang, 2010) and can be entrained to external rhythms (Rees et al., 1986; Regan, 1966). As such, neural oscillations have been hypothesized as natural pacemakers for conscious time estimation (Buhusi and Meck, 2005; Pöppel, 1997; Treisman et al., 1990; Varela et al., 1981). However, within this framework, a major problem for the brain is to determine when events occur with respect to its internal frame of reference. Our results suggest that the timing of events could automatically be encoded in the phase of a recruited pacemaker or entrained oscillation (thereby acting as a temporal frame of reference for cortex) and that the variation of the pacemaker's phase over time results in variation of perceived timing.

\section{A canonical role for the phase of intrinsic and entrained neural oscillations?}

It is noteworthy that we specifically targeted the delta range using neural entrainment or frequency-tagging. EEG studies have previously tested the idea that the order of visual events was coded in the phase of the alpha oscillatory component (Gho and Varela, 1988) and recent studies have pointed out to the role of theta/alpha in temporal visual illusions (Chakravarthi and Vanrullen, 2012; VanRullen et al., 2006).
These studies suggest that intrinsic oscillations are recruited for the encoding of events in the absence of external temporal regularities. Recent hypotheses further extend the notion that the phase of lowfrequency neural oscillations is crucial for the encoding of order - for instance with the implication of the theta band in working memory (Lisman and Jensen, 2013) - or even for temporal parsing - for instance, in speech (Giraud and Poeppel, 2012). The temporal encoding mechanisms described in our experiment are de facto constrained by the rhythmicity of the external inputs; in turn, however, the encoding of event timing may capitalize on the temporal features provided by external stimulation to build a temporal reference frame or pacemaker consistent with the rhythms provided by the external sensory world. While delta oscillations have been previously linked to temporal predictability (Stefanics et al., 2010), further investigations need to be done to test their implication in the encoding of event timing when no rhythmic stimulation or external temporal reference frame is provided.

\section{Evoked activity and attention to time}

Latency-based descriptions of cognitive functions classically use event-related potentials/fields (ERP and ERF, respectively) to describe the timing at which mental operations take place in cortex (Coles and Rugg, 1995; Madl et al., 2011). The auditory and visual evoked responses were thus expected to partly reflect participants' perceptual shifts in AV simultaneity taking place during lag-adaptation. However, 
A

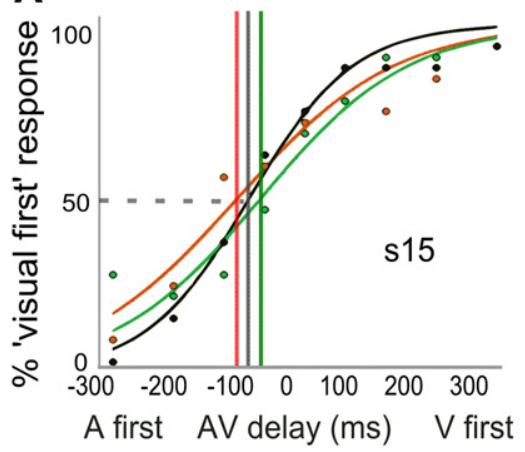

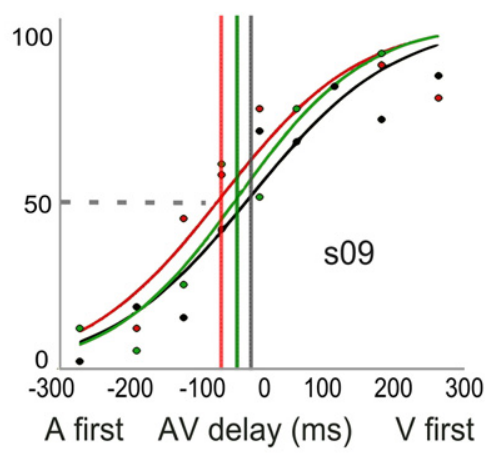

B

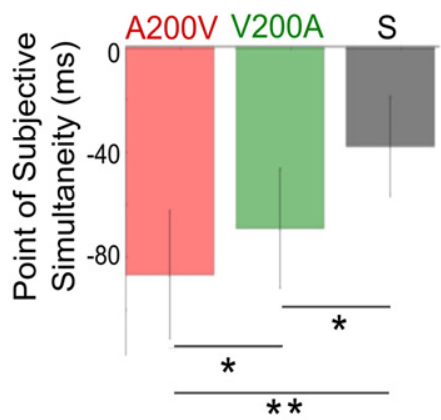

C

Subject

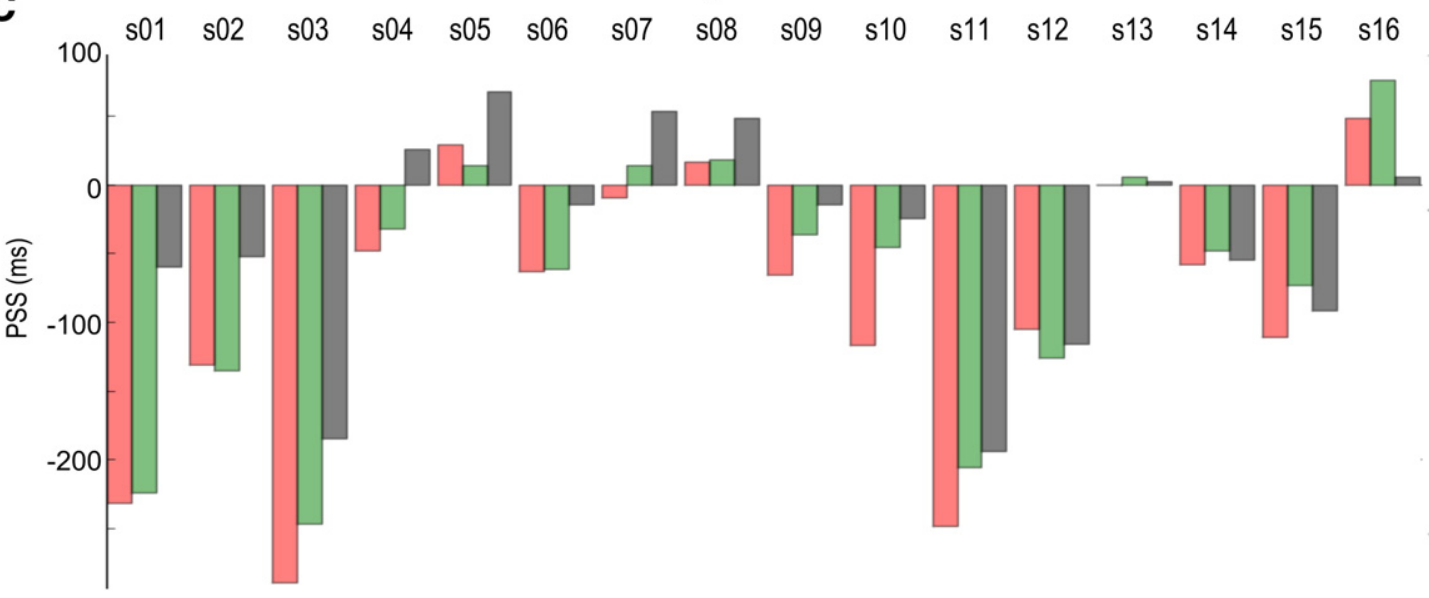

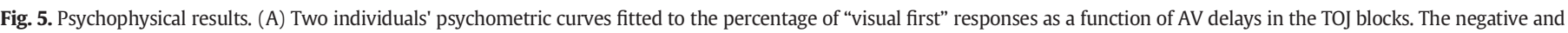

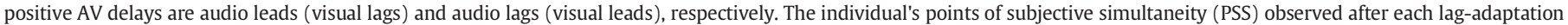

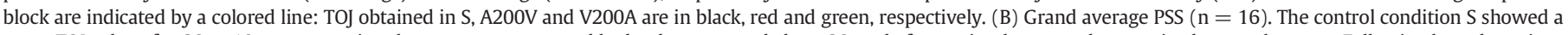

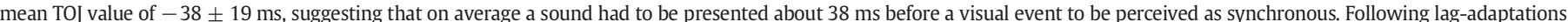

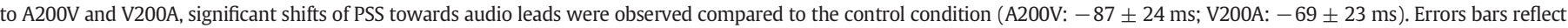

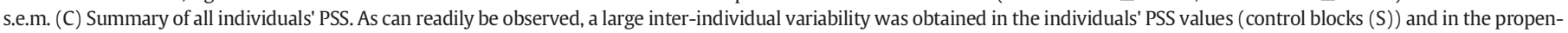
sity of a given individual to temporally shift his or her natural PSS.

and surprisingly, no significant changes in the amplitude or in the latency of the evoked responses were observed in the course of lagadaptation, albeit clear visual modulations of the auditory responses were seen. Previous EEG studies using auditory and tactile stimuli during a TOJ task reported amplitude modulation (McDonald et al., 2005) or latency shifts (Vibell et al., 2007) of the evoked sensory responses as a function of which sensory modality was attended. It was notably reported that attention could speed up the processing of the attended sensory modality. Here, no systematic changes in the evoked profiles were observed suggesting that during lag-adaptation participants equally paid attention to the auditory and visual events as per task requirements (cf. methods).

Although the lack of significant lag-adaptation suppression (GrillSpector et al., 2006) of the evoked responses was surprising, it is classically known that attention can attenuate the effect of neural suppression (Gazzaley et al., 2005). Here, participants were asked to pay attention to any deviants presented in the auditory, visual or audiovisual modalities during lag-adaptation. The lack of repetition suppression in both sensory cortices may thus be an index of successful attentional orienting. Additionally, recent findings have shown that the more temporally predictable, the higher the repetition suppression effects notably in the auditory responses (Costa-Faidella et al., 2011; Summerfield et al., 2008, 2011). In the context of predictive coding models, it has also been suggested that repetition and expectation were dissociable (Todorovic and de Lange, 2012). The current experimental design did not allow us to dissociate the factor of predictability and expectation but these observations provide an alternative speculation, namely that paying attention to time may alleviate neural lag-adaptation.

One relevant point here is the integrative vs. segregative nature of the task with regard to multisensory processing: experimental paradigms using multisensory integration have classically reported an increase of sensory evoked responses, for instance when using the sound-induced flash illusion (Mishra et al., 2007; Watkins et al., 2006). In a TOJ task however, the segregation of auditory and visual information is a pre-requisite for successful ordering of auditory and visual events in time. Participants were repeatedly presented with AV lags at an entrainment rate consistent with automatic multisensory integration (Kösem and van Wassenhove, 2012): as such, audiovisual binding was reinforced in this task and an increased evoked response would have been expected. However, and at the same time, a decrease of the evoked responses was expected by virtue of neural suppression (Grill-Spector et al., 2006). Hence, one possible explanation for the absence of significant modulations of the sensory evoked responses in the course of adaptation is the competition between the integrative and segregative processes in this particular experimental design. Additional work will be required to further address this working hypothesis.

Phase of neural oscillations: encoding time (or space?)

Multisensory integration is known to capitalize on the spatiotemporal coincidence of sensory events (Colonius and Diederich, 2010; Meredith et al., 1987) and visual capture of auditory spatial representation is a 

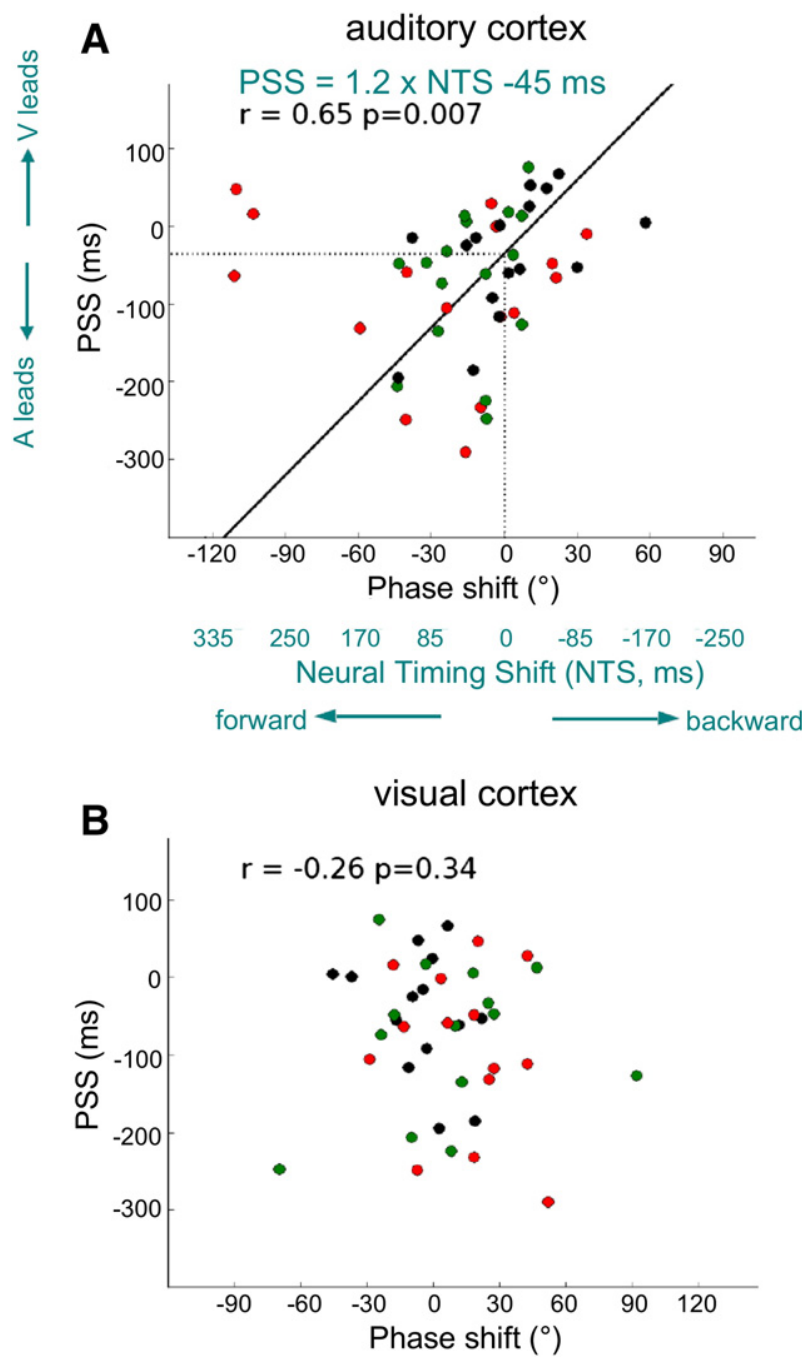

Fig. 6. Phase shifts reflect subjective timing. Individuals' PSS were plotted as a function of the difference in mean instantaneous phase (end minus beginning in a given lagadaptation period) in auditory (A) and visual cortices (B). Each data point corresponds to an individual duplet, namely: the mean phase difference obtained in a given lagadaptation period (S: black; A200V: red; V200A: green) and the associated individual's PSS measured during the following TOJ block. A linear regression was computed on a per individual basis between the mean PSS and the circular mean of the instantaneous phases of the $1 \mathrm{~Hz}$ neural oscillation obtained across lag-adaptation blocks. A significant correlation was found between the phase shifts of the entrained $1 \mathrm{~Hz}$ neural oscillation in auditory cortices and individuals' PSS $(r=0.65, \mathrm{p}<0.01)$ whereas no such correlation was found in visual cortices $(r=-0.26$, n.s.).

classic phenomenon (i.e. ventriloquism,(Alais and Burr, 2004; Lewald and Guski, 2003; Slutsky and Recanzone, 2001)) well accounted for by Bayesian models of multisensory integration (Alais and Burr, 2004; Burr and Alais, 2006; Ernst and Bülthoff, 2004; Witten and Knudsen, 2005). More generally, vision tends to be most reliable in encoding spatial cues whereas audition provides the most reliable temporal cues. In the experimental design used here, visual events were displayed at a constant distance on the monitor screen whereas sounds were presented via headphones. By virtue of spatiotemporal coincidence and given the consistent AV timing over lag-adaptation, the auditory distance would have to be adjusted to visual information. In a scheme analogous to the calibration of auditory spatial representation by vision in the barn owl (Knudsen and Brainard, 1991), the observed non-stationary phase shifts in auditory cortices could thus reflect an automatic means to fine tune spatiotemporal coincidence across sensory modalities. Specifically, auditory spatial uncertainty could be compensated for by the stable spatiotemporal reference frame established in vision. From this viewpoint, the auditory system would not act as a timer per se; rather, the distance of auditory events would be actively made compatible with visual inputs to form an integrated AV percept. Hence, shifts in AV simultaneity may reflect the compensation of temporal delays in audition. Such mechanism would predict what we observed, namely that the entrainment of auditory and visual cortices is asymmetrical when attention is directed to the timing of events (i.e. to the dominant sensory modality for timing, namely audition); it also predicts that response times for audition (but not for vision) vary during AV delay exposure (Navarra et al., 2009) as well as the correlation between reaction times and visual to auditory phasereset previously described (Thorne et al., 2011). Our results further support a recent discussion on the functional asymmetry between the sampling of acoustic and visual information over time (Thorne et al., 2011), namely: while the visual system may naturally rely on endogenous rhythms (e.g. alpha oscillations, Gho and Varela, 1988; Jensen et al., 2012; VanRullen and Koch, 2003; Varela et al., 1981) and overt sampling (e.g. (micro)saccades, Schroeder and Lakatos, 2009), the auditory system may necessitate temporal-locking to incoming acoustic inputs to accurately represent information over time (Giraud and Poeppel, 2012; Henry and Obleser, 2012; Stefanics et al., 2010; Thorne et al., 2011). Hence, while visual timing may rely on an internally generated temporal reference frame, audition may require the establishment of a temporal reference frame on the go and locked to the temporal statistics of the auditory environment.

\section{Neural oscillations: multiplex encoding of information}

Our results suggest that, in cortex, the phase of neural oscillations may provide an automatic means to flag events in a brain's referential time - i.e. provide the needed brain-centric view of time (Scharnowski et al., 2013). The encoding of spatiotemporal information in the phase of neural oscillations has been described in the hippocampus in which mechanisms of phase precession encode spatial locations as the animal navigates in a maze (Buzsáki, 2002; Lisman, 2005; Skaggs et al., 1995). Phase precession mechanisms may not be exclusive to hippocampal networks nor to spatial processing and may serve a more general purpose such as encoding events for working memory while preserving temporal order (Lisman, 2005; Lisman and Jensen, 2013). In particular, the content of a sensory event is encoded by the neural assembly firing within a certain gamma cycle (Lisman, 2005; Lisman and Jensen, 2013) while the relative timing of the event is encoded in the phase of the theta oscillation (Lisman, 2005; Lisman and Jensen, 2013).

In cortex, low-frequency neural oscillations are known to regulate the excitability of neural ensembles such that specific phases of lowfrequency neural oscillations are associated with periods of high and low neuronal excitability (Buzsáki, 2010; Lakatos et al., 2008; Schroeder and Lakatos, 2009): the phase of low-frequency neural oscillations modulates the power of high-frequency neural oscillatory responses, a mechanism known as phase-power or cross-frequency coupling (Canolty et al., 2006). Neural synchronizations in higher frequency ranges (e.g. gamma range, $>40 \mathrm{~Hz}$ ) provide a reliable index of feature binding within and across sensory modalities (Arnal et al., 2011; Engel et al., 1991; Roelfsema et al., 1997; Senkowski et al., 2008; Tallon-Baudry and Bertrand, 1999). In multisensory integration, low-frequency neural oscillations (delta, $1-2 \mathrm{~Hz}$ ) play a crucial role in the temporal selection (Besle et al., 2011; Fiebelkorn et al., 2013; Gomez-Ramirez et al., 2011; Lakatos et al., 2008; Schroeder and Lakatos, 2009) and in the integration of AV information (Fiebelkorn et al., 2011; Kösem and van Wassenhove, 2012; Luo et al., 2010).

Hence, the phase of low-frequency oscillations may provide the finegrained temporal resolution needed for the segregation of AV event timing and conscious timing while preserving integration processes through neural synchronization (necessary in building the mental representation of a multisensory AV object).

In such scheme, the informational chunking operates over an oscillatory cycle by eliciting temporal windows of high neural excitability for 
integration (Panzeri et al., 2010; van Wassenhove, 2009), while the phase of neural oscillations provides the temporal stamping operation needed to preserve the timing of operations in parallel systems. As such, the same informational content can be encoded in a multiplexed manner with (i) integration operating on those sensory attributes used in the building of an internal object (Engel and Singer, 2001; Treisman, 1996) while (ii) segregation - or temporal stamping provides the automatic encoding of event timing. Such temporal encoding framework comes in support of intrinsic and non-dedicated models of time perception over small time scales (Karmarkar and Buonomano, 2007). Our data further converge with recent findings showing that accurate phase encoding of the temporal structure of sensory events affords predictability (Schroeder and Lakatos, 2009; Stefanics et al., 2010) and support recent phase-coding approaches in computational neurosciences (Nadasdy, 2010).

\section{Conclusion}

We showed that perceived simultaneity linearly maps onto the phase of neural oscillations in the auditory cortex. Our findings complement recent findings showing that accurate phase encoding of temporal event structure affords predictability (Schroeder and Lakatos, 2009; Stefanics et al., 2010) and enhances task performance (Busch et al., 2009; Monto et al., 2008; Neuling et al., 2012; Romei et al., 2012; Varela et al., 1981). Our results further suggest that mechanisms analogous to phase precession in the hippocampus may be used in cortex for the encoding of event timing. Specifically, the phase of slow oscillatory activity in sensory areas may provide a canonical means to organize sensory inputs in time. Future work will address the possibility that a canonical function of neural oscillations is the encoding of event timing serving the emergence of psychological time.

Supplementary data to this article can be found online at http://dx. doi.org/10.1016/j.neuroimage.2014.02.010.

\section{Acknowledgments}

This work was supported by an ERC-YStG-263584, an ANR10JCJC1904 and an IRG-249222 to V.vW. We are grateful to the NeuroSpin infrastructure groups, for their support in participant recruitment; and to anonymous reviewers for their comments on prior version of the manuscript.

\section{Conflict of interest}

The authors report no conflict of interest.

\section{References}

Alais, D., Burr, D., 2004. The ventriloquist effect results from near-optimal bimodal integration. Curr. Biol. 14, 257-262.

Arnal, L.H., Wyart, V., Giraud, A.-L., 2011. Transitions in neural oscillations reflect prediction errors generated in audiovisual speech. Nat. Neurosci. 14, 797-801.

Besle, J., Schevon, C.A., Mehta, A.D., Lakatos, P., Goodman, R.R., McKhann, G.M., Emerson, R.G., Schroeder, C.E., 2011. Tuning of the human neocortex to the temporal dynamics of attended events. J. Neurosci. 31, 3176-3185.

Brainard, D.H., 1997. The Psychophysics toolbox. Spat. Vis. 10, 433-436.

Buhusi, C.V., Meck, W.H., 2005. What makes us tick? Functional and neural mechanisms of interval timing. Nat. Rev. Neurosci. 6, 755-765.

Buhusi, C.V., Meck, W.H., 2009. Relativity theory and time perception: single or multiple clocks? PLoS One 4, e6268.

Burr, D., Alais, D., 2006. Combining visual and auditory information. Prog. Brain Res. 155, 243-258.

Busch, N.A., Dubois, J., VanRullen, R., 2009. The phase of ongoing EEG oscillations predicts visual perception. J. Neurosci. 29, 7869-7876.

Buzsáki, G., 2002. Theta oscillations in the hippocampus. Neuron 33, 325-340.

Buzsáki, G., 2010. Neural syntax: cell assemblies, synapsembles, and readers. Neuron 68, 362-385.

Buzsáki, G., Draguhn, A., 2004. Neuronal oscillations in cortical networks. Science 304, 1926-1929.

Cai, M., Stetson, C., Eagleman, D.M., 2012. A neural model for temporal order judgments and their active recalibration: a common mechanism for space and time? Front. Psychol. 3, 470.
Canolty, R.T., Edwards, E., Dalal, S.S., Soltani, M., Nagarajan, S.S., Berger, M.S., Barbaro, N.M., Knight, R.T., 2006. High gamma power is phase-locked to theta oscillations in human neocortex. Science 313, 1626-1628.

Capilla, A., Pazo-Alvarez, P., Darriba, A., Campo, P., Gross, J., 2011. Steady-state visual evoked potentials can be explained by temporal superposition of transient eventrelated responses. PLoS One 6.

Chakravarthi, R., Vanrullen, R., 2012. Conscious updating is a rhythmic process. Proc. Natl. Acad. Sci. U. S. A. 109, 10599-10604

Chopin, A., Mamassian, P., 2012. Predictive properties of visual adaptation. Curr. Biol. 22, 622-626.

Church, R.M., 1984. Properties of the internal clock. Ann. N. Y. Acad. Sci. 423, 566-582.

Coles, M., Rugg, M., 1995. Event-Related Brain Potentials: An Introduction. In: Coles, M., Rugg, M. (Eds.), Electrophysiology of Mind: Event-Related Brain Potentials and Cognition. Oxford University Press, London, pp. 1-26.

Colonius, H., Diederich, A., 2010. The optimal time window of visual-auditory integration: a reaction time analysis. Front. Integr. Neurosci. 4, 11.

Costa-Faidella, J., Baldeweg, T., Grimm, S., Escera, C., 2011. Interactions between "what" and "when" in the auditory system: temporal predictability enhances repetition suppression. J. Neurosci. 31, 18590-18597.

Coull, J.T., Vidal, F., Nazarian, B., Macar, F., 2004. Functional anatomy of the attentional modulation of time estimation. Science 303, 1506-1508.

Dale, A.M., Fischl, B., Sereno, M.I., 1999. Cortical surface-based analysis. I. Segmentation and surface reconstruction. Neurolmage 9, 179-194.

Engel, A.K., Singer, W., 2001. Temporal binding and the neural correlates of sensory awareness. Trends Cogn. Sci. 5, 16-25.

Engel, A.K., König, P., Singer, W., 1991. Direct physiological evidence for scene segmentation by temporal coding. Proc. Natl. Acad. Sci. U. S. A. 88, 9136-9140.

Ernst, M.O., Bülthoff, H.H., 2004. Merging the senses into a robust percept. Trends Cogn. Sci. 8, 162-169.

Fiebelkorn, I.C., Foxe, J.J., Butler, J.S., Mercier, M.R., Snyder, A.C., Molholm, S., 2011. Ready, set, reset: stimulus-locked periodicity in behavioral performance demonstrates the consequences of cross-sensory phase reset. J. Neurosci. 31, 9971-9981.

Fiebelkorn, I.C., Snyder, A.C., Mercier, M.R., Butler, J.S., Molholm, S., Foxe, J.J., 2013. Cortical cross-frequency coupling predicts perceptual outcomes. NeuroImage 69, 126-137.

Fischl, B., Dale, A.M., 2000. Measuring the thickness of the human cerebral cortex from magnetic resonance images. Proc. Natl. Acad. Sci. U. S. A. 97, 11050-11055.

Fisher, N.I., 1995. Statistical Analysis of Circular Data. Cambridge University Press, Cambridge.

Fujisaki, W., Shimojo, S., Kashino, M., Nishida, S., 2004. Recalibration of audiovisual simultaneity. Nat. Neurosci. 7, 773-778.

Gazzaley, A., Cooney, J.W., Rissman, J., D'Esposito, M., 2005. Top-down suppression deficit underlies working memory impairment in normal aging. Nat. Neurosci. 8, $1298-1300$

Gho, M., Varela, F.J., 1988. A quantitative assessment of the dependency of the visual temporal frame upon the cortical rhythm. J. Physiol. Paris 83, 95-101.

Giraud, A.-L., Poeppel, D., 2012. Cortical oscillations and speech processing: emerging computational principles and operations. Nat. Neurosci. 15, 511-517.

Gomez-Ramirez, M., Kelly, S.P., Molholm, S., Sehatpour, P., Schwartz, T.H., Foxe, J.J., 2011. Oscillatory sensory selection mechanisms during intersensory attention to rhythmic auditory and visual inputs: a human electrocorticographic investigation. J. Neurosci. 31, 18556-18567.

Gramfort, A., Luessi, M., Larson, E., Engemann, D.A., Strohmeier, D., Brodbeck, C., Goj, R., Jas, M., Brooks, T., Parkkonen, L., Hämäläinen, M., 2013. MEG and EEG data analysis with MNE-python. Front. Neurosci. 7.

Gramfort, A., Luessi, M., Larson, E., Engemann, D.A., Strohmeier, D., Brodbeck, C., Parkkonen, L., Hämäläinen, M.S., 2014. MNE software for processing MEG and EEG data. NeuroImage 86, 446-460.

Grill-Spector, K., Henson, R., Martin, A., 2006. Repetition and the brain: neural models of stimulus-specific effects. Trends Cogn. Sci. 10, 14-23.

Gross, J., Baillet, S., Barnes, G.R., Henson, R.N., Hillebrand, A., Jensen, O., Jerbi, K., Litvak, V., Maess, B., Oostenveld, R., Parkkonen, L., Taylor, J.R., van Wassenhove, V., Wibral, M., Schoffelen, J.-M., 2013. Good practice for conducting and reporting MEG research. NeuroImage 65, 349-363.

Hämäläinen, M.S., Sarvas, J., 1989. Realistic conductivity geometry model of the human head for interpretation of neuromagnetic data. IEEE Trans. Biomed. Eng. 36, 165-171.

Harrington, D.L., Haaland, K.Y., Knight, R.T., 1998. Cortical networks underlying mechanisms of time perception. J. Neurosci. 18, 1085-1095.

Henry, M.J., Obleser, J., 2012. Frequency modulation entrains slow neural oscillations and optimizes human listening behavior. Proc. Natl. Acad. Sci. U. S. A. 109, 20095-20100.

Heron, J., Roach, N.W., Whitaker, D., Hanson, J.V.M., 2010. Attention regulates the plasticity of multisensory timing. Eur. J. Neurosci. 31, 1755-1762.

Ivry, R.B., Schlerf, J.E., 2008. Dedicated and intrinsic models of time perception. Trends Cogn. Sci. 12, 273-280.

Jensen, O., Bonnefond, M., VanRullen, R., 2012. An oscillatory mechanism for prioritizing salient unattended stimuli. Trends Cogn. Sci. 16, 200-206.

Johnston, A., Nishida, S., 2001. Time perception: brain time or event time? Curr. Biol. 11, R427-R430.

Karmarkar, U.R., Buonomano, D.V., 2007. Timing in the absence of clocks: encoding time in neural network states. Neuron 53, 427-438.

Keil, A., Debener, S., Gratton, G., Junghöfer, M., Kappenman, E.S., Luck, S.J., Luu, P., Miller, G.A., Yee, C.M., 2013. Committee report: publication guidelines and recommendations for studies using electroencephalography and magnetoencephalography. Psychophysiology 51, 1-21.

Knudsen, E., Brainard, M., 1991. Visual instruction of the neural map of auditory space in the developing optic tectum. Science $253,85-87$. 
Kösem, A., van Wassenhove, V., 2012. Temporal structure in audiovisual sensory selection. PLoS One 7, e40936.

Lachaux, J.P., Rodriguez, E., Martinerie, J., Varela, F.J., 1999. Measuring phase synchrony in brain signals. Hum. Brain Mapp. 8, 194-208.

Lakatos, P., Karmos, G., Mehta, A.D., Ulbert, I., Schroeder, C.E., 2008. Entrainment of neuronal oscillations as a mechanism of attentional selection. Science 320, 110-113.

Lewald, J., Guski, R., 2003. Cross-modal perceptual integration of spatially and temporally disparate auditory and visual stimuli. Cogn. Brain Res. 16, 468-478.

Lin, F.-H., Belliveau, J.W., Dale, A.M., Hämäläinen, M.S., 2006. Distributed current estimates using cortical orientation constraints. Hum. Brain Mapp. 27, 1-13.

Lisman, J., 2005. The theta/gamma discrete phase code occurring during the hippocampal phase precession may be a more general brain coding scheme. Hippocampus 15 913-922.

Lisman, J.E., Jensen, O., 2013. The theta-gamma neural code. Neuron 77, 1002-1016.

Love, S.A., Petrini, K., Cheng, A., Pollick, F.E., 2013. A psychophysical investigation of differences between synchrony and temporal order judgments. PLoS One 8, e54798.

Luo, H., Liu, Z., Poeppel, D., 2010. Auditory cortex tracks both auditory and visual stimulus dynamics using low-frequency neuronal phase modulation. PLoS Biol. 8, e1000445.

Madl, T., Baars, B.J., Franklin, S., 2011. The timing of the cognitive cycle. PLoS One 6, e14803.

Maris, E., Oostenveld, R., 2007. Nonparametric statistical testing of EEG- and MEG-data. J. Neurosci. Methods 164, 177-190.

McDonald, J.J., Teder-Sälejärvi, W.A., Di Russo, F., Hillyard, S.A., 2005. Neural basis of auditory-induced shifts in visual time-order perception. Nat. Neurosci. 8, 1197-1202.

Meredith, M., Nemitz, J., Stein, B., 1987. Determinants of multisensory integration in superior colliculus neurons. I. Temporal factors. J. Neurosci. 7, 3215-3229.

Mishra, J., Martinez, A., Sejnowski, T.J., Hillyard, S.A., 2007. Early cross-modal interactions in auditory and visual cortex underlie a sound-induced visual illusion. J. Neurosci. 27, 4120-4131.

Miyazaki, M., Yamamoto, S., Uchida, S., Kitazawa, S., 2006. Bayesian calibration of simultaneity in tactile temporal order judgment. Nat. Neurosci. 9, 875-877.

Monto, S., Palva, S., Voipio, J., Palva, J.M., 2008. Very slow EEG fluctuations predict the dynamics of stimulus detection and oscillation amplitudes in humans. J. Neurosci. 28 , 8268-8272.

Morillon, B., Kell, C.A., Giraud, A.-L., 2009. Three stages and four neural systems in time estimation. J. Neurosci. 29, 14803-14811.

Moutoussis, K., Zeki, S., 1997. A direct demonstration of perceptual asynchrony in vision. Proc. Biol. Sci. 264, 393-399.

Nadasdy, Z., 2010. Binding by asynchrony: the neuronal phase code. Front. Neurosci. 4.

Navarra, J., Hartcher-O'Brien, J., Piazza, E., Spence, C., 2009. Adaptation to audiovisual asynchrony modulates the speeded detection of sound. Proc. Natl. Acad. Sci. U. S. A. 106, 9169-9173.

Neuling, T., Rach, S., Wagner, S., Wolters, C.H., Herrmann, C.S., 2012. Good vibrations: oscillatory phase shapes perception. NeuroImage 63, 771-778.

Nozaradan, S., Peretz, I., Missal, M., Mouraux, A., 2011. Tagging the neuronal entrainment to beat and meter. J. Neurosci. 31, 10234-10240.

Panzeri, S., Brunel, N., Logothetis, N.K., Kayser, C., 2010. Sensory neural codes using multiplexed temporal scales. Trends Neurosci. 33, 111-120.

Pöppel, E., 1997. A hierarchical model of temporal perception. Trends Cogn. Sci. 1, 56-61.

Rees, A., Green, G.G.R., Kay, R.H., 1986. Steady-state evoked responses to sinusoidally amplitude-modulated sounds recorded in man. Hear. Res. 23, 123-133.

Regan, D., 1966. Some characteristics of average steady-state and transient responses evoked by modulated light. Electroencephalogr. Clin. Neurophysiol. 20, 238-248.

Roelfsema, P., Engel, A., Konig, P., Singer, W., 1997. Visuomotor integration is associated with zero time-lag synchronization among cortical areas. Nature 385, 157-161.

Romei, V., Gross, J., Thut, G., 2012. Sounds reset rhythms of visual cortex and corresponding human visual perception. Curr. Biol. 22, 807-813.

Roopun, A.K., Kramer, M.A., Carracedo, L.M., Kaiser, M., Davies, C.H., Traub, R.D., Kopell, N.J., Whittington, M.A., 2008. Temporal interactions between cortical rhythms. Front. Neurosci. 2, 145-154.

Scharnowski, F., Rees, G., Walsh, V., 2013. Time and the brain: neurorelativity: the chronoarchitecture of the brain from the neuronal rather than the observer's perspective. Trends Cogn. Sci. 17, 51-52.

Schroeder, C.E., Lakatos, P., 2009. Low-frequency neuronal oscillations as instruments of sensory selection. Trends Neurosci. 32, 9-18.

Senkowski, D., Schneider, T.R., Foxe, J.J., Engel, A.K., 2008. Crossmodal binding through neural coherence: implications for multisensory processing. Trends Neurosci. 31, 401-409.

Shaw, M.E., Hämäläinen, M.S., Gutschalk, A., 2013. How anatomical asymmetry of human auditory cortex can lead to a rightward bias in auditory evoked fields. Neurolmage $74,22-29$.
Skaggs, W.E., Knierim, J.J., Kudrimoti, H.S., McNaughton, B.L., 1995. A model of the neura basis of the rat's sense of direction. Adv. Neural Inf. Process. Syst. 7, 173-180.

Slutsky, D.A., Recanzone, G.H., 2001. Temporal and spatial dependency of the ventriloquism effect. Neuroreport 12, 7-10.

Stefanics, G., Hangya, B., Hernádi, I., Winkler, I., Lakatos, P., Ulbert, I., 2010. Phase entrainment of human delta oscillations can mediate the effects of expectation on reaction speed. J. Neurosci. 30, 13578-13585.

Stone, J.V., Hunkin, N.M., Porrill, J., Wood, R., Keeler, V., Beanland, M., Port, M., Porter, N.R. 2001. When is now? Perception of simultaneity. Proc. Biol. Sci. 268, 31-38.

Sugita, Y., Suzuki, Y., 2003. Implicit estimation of sound-arrival time. Nature 421.

Summerfield, C., Trittschuh, E.H., Monti, J.M., Mesulam, M.M., Egner, T., 2008. Neural repetition suppression reflects fulfilled perceptual expectations. Nat. Neurosci. 11, 1004-1006.

Summerfield, C., Wyart, V., Johnen, V.M., de Gardelle, V., 2011. Human Scalp electroencephalography reveals that repetition suppression varies with expectation. Front. Hum. Neurosci. 5, 67.

Tallon-Baudry, C., Bertrand, O., 1999. Oscillatory gamma activity in humans and its role in object representation. Trends Cogn. Sci. 3, 151-162.

Taulu, S., Kajola, M., Simola, J., 2003. Suppression of interference and artifacts by the signal space separation method. Brain Topogr. 16, 269-275.

Thorne, J.D., De Vos, M., Viola, F.C., Debener, S., 2011. Cross-modal phase reset predicts auditory task performance in humans. J. Neurosci. 31, 3853-3861.

Todorovic, A., de Lange, F.P., 2012. Repetition suppression and expectation suppression are dissociable in time in early auditory evoked fields. J. Neurosci. 32, 13389-13395.

Treisman, M., 1963. Temporal discrimination and the indifference interval: Implications for a model of the "internal clock". Psychol. Monogr. Gen. Appl. 77, 1.

Treisman, M., 1984. Temporal rhythms and cerebral rhythms. Ann. N. Y. Acad. Sci. 423, 542-565.

Treisman, A., 1996. The binding problem. Curr. Opin. Neurobiol. 6, 171-178.

Treisman, M., Faulkner, A., Naish, P.L., Brogan, D., 1990. The internal clock: evidence for a temporal oscillator underlying time perception with some estimates of its characteristic frequency. Perception 19, 705-743.

Treisman, M., Faulkner, A., Naish, P.L.N., 1992. On the relation between time perception and the timing of motor action: evidence for a temporal oscillator controlling the timing of movement. Q. J. Exp. Psychol. A 45, 235-263.

Uusitalo, M.A., Ilmoniemi, R.J., 1997. Signal-space projection method for separating MEG or EEG into components. Med. Biol. Eng. Comput. 35, 135-140.

Van Eijk, R.L.J., Kolhlrausch, A., Juola, J.F., Van de Par, S., 2008. Audiovisual synchrony and temporal order judgments: effects of experimental method and stimulus type. Percept. Psychophys. 70, 955-968.

Van Wassenhove, V., 2009. Minding time in an amodal representational space. Philos. Trans. R. Soc. Lond. B Biol. Sci. 364, 1815-1830.

VanRullen, R., Koch, C., 2003. Is perception discrete or continuous? Trends Cogn. Sci. 7, 207-213.

VanRullen, R., Reddy, L., Koch, C., 2006. The continuous wagon wheel illusion is associated with changes in electroencephalogram power at approximately $13 \mathrm{~Hz}$. J. Neurosci. 26, 502-507.

Varela, F., Toro, A., John, E.R., Schwartz, E., 1981. Perceptual framing and cortical alpha rhythm. Neuropsychologia 19, 675-686.

Vibell, J., Klinge, C., Zampini, M., Spence, C., Nobre, A.C., 2007. Temporal order is coded temporally in the brain: early event-related potential latency shifts underlying prior entry in a cross-modal temporal order judgment task. J. Cogn. Neurosci. 19 109-120.

Vroomen, J., Keetels, M., 2010. Perception of intersensory synchrony: a tutorial review. Atten. Percept. Psychophys. 72, 871-884.

Vroomen, J., Keetels, M., de Gelder, B., Bertelson, P., 2004. Recalibration of temporal order perception by exposure to audio-visual asynchrony. Brain Res. Cogn. Brain Res. 22, 32-35.

Wang, X., 2010. Neurophysiological and computational principles of cortical rhythms in cognition. Phys. Rev. 90, 1195-1268.

Watkins, S., Shams, L., Tanaka, S., Haynes, J.-D., Rees, G., 2006. Sound alters activity in human V1 in association with illusory visual perception. Neurolmage 31, 1247-1256.

Witten, I.B., Knudsen, E.I., 2005. Why seeing is believing: merging auditory and visua worlds. Neuron 48, 489-496.

Wittmann, M., 2009. The inner experience of time. Philos. Trans. R. Soc. Lond. B Biol. Sci. 364, 1955-1967.

Wittmann, M., 2013. The inner sense of time: how the brain creates a representation of duration. Nat. Rev. Neurosci. 14, 217-223.

Yamamoto, S., Miyazaki, M., Iwano, T., Kitazawa, S., 2012. Bayesian calibration of simultaneity in audiovisual temporal order judgments. PLoS One 7, e40379.

Zeki, S., Bartels, A., 1998. The asynchrony of consciousness. Proc. Biol. Sci. 265, 1583-1585. 